\title{
Modular Entanglement
}

\author{
Giulia Gualdi, Salvatore M. Giampaolo, and Fabrizio Illuminati \\ Dipartimento di Matematica e Informatica, Università degli Studi di Salerno, \\ Via Ponte don Melillo, I-84084 Fisciano (SA), Italy; CNR-SPIN, \\ and INFN Sezione di Napoli, Gruppo collegato di Salerno, I-84084 Fisciano (SA), Italy
}

(Dated: November 7, 2010)

\begin{abstract}
We introduce and discuss the concept of modular entanglement. This is the entanglement that is established between the end points of modular systems composed by sets of interacting moduli of arbitrarily fixed size. We show that end-to-end modular entanglement scales in the thermodynamic limit and rapidly saturates with the number of constituent moduli. We clarify the mechanisms underlying the onset of entanglement between distant and non-interacting quantum systems and its optimization for applications to quantum repeaters and entanglement distribution and sharing.
\end{abstract}

PACS numbers: 03.67.Mn,03.67.Bg,03.67.Hk,75.10.Pq

Introduction: Entanglement generation and distribution over long distances is crucial to the realization of large-scale quantum computation and information tasks, including teleportation, memories and repeaters, and secure key distribution [1]. Unfortunately, in systems with short-range interactions bipartite entanglement decreases rapidly with distance 2], whereas in systems with longrange interactions entanglement monogamy prevents the creation of strongly entangled pairs [3]. Systems with finite correlation length, such as the 1-D Heisenberg and $X X$ models, allow for sizeable ground-state end-to-end entanglement, independent of the size of the system, provided that simple patterns of site-dependent couplings are selected. This phenomenon has been termed LongDistance Entanglement (LDE) [4]. It can be optimized against thermal decoherence in models with more sophisticated patterns of couplings [5], and might be implemented using suitably engineered atom-optical systems, including optical lattices and coupled cavity arrays 5, 6]. However, its thermal instability remains a paramount obstacle against possible implementations.

In the present work we consider the general problem of generating and distributing entanglement between distant and non-interacting quantum systems. We discuss the properties of renormalization, amplification, and scalability of end-to-end entanglement in quantum manybody systems. From this investigation there emerges naturally the concept of Modular Entanglement. Specifically, we will show that size-independent end-to-end entanglement arises in the ground state of modular systems constituted by a set of identically interacting moduli of arbitrarily fixed size. This general type of entanglement at long distances, termed Modular Entanglement (ME), includes LDE as the particular case realized in systems formed of two-qubit moduli.

We will show that a most relevant feature of ME is its enhanced stability against thermal decoherence, even by several orders of magnitude, compared to the case of simple LDE. Indeed, as we will discuss, genuine ME is generated also in systems composed by moduli that, individ- ually, do not exhibit sizeable end-to-end entanglement. This is in sharp contrast with systems featuring LDE, for which the individual moduli always exhibit maximal end-to-end entanglement. Besides showing that LDE is a special, singular case of the much more general ME, these findings imply that end-to-end entanglement at large distances is an emerging collective property that need not be shared by the individual constituents.

We will investigate first the onset of ME in minimal modular systems made of two identically interacting moduli. We will then consider many-moduli systems and discuss the mechanisms associated to the onset of ME and its scaling behaviour with the number of moduli.

Two moduli: Here and throughout the paper we will consider 1-D systems made of $N$ moduli each containing $n$ spins, such that the total number of spins in the system is $n_{t}=n N$. We first consider the most elementary modular system composed of $N=2$ moduli $B_{1}$ and $B_{2}$ (See Fig. 1. upper panel), endowed with site-dependent, isotropic, nearest-neighbor exchange interactions. The total Hamiltonian of the system reads:

$$
H_{T, 2 \operatorname{moduli}}=H_{1, n}^{B_{1}}+H_{n+1,2 n}^{B_{2}}+H_{n, n+1}^{I}
$$

with

$$
H_{\gamma, \delta}^{\alpha}=\frac{1}{2} \sum_{i=\gamma}^{\delta-1} J_{i, i+1}\left(S_{i}^{x} S_{i+1}^{x}+S_{i}^{y} S_{i+1}^{y}\right),
$$

where $H_{n, n+1}^{I}$ is the interaction Hamiltonian between the two boundary qubits in $B_{1}$ and $B_{2}$. We now investigate how and to what extent the generation of end-to-end bipartite entanglement in a modular system depends on the entanglement properties of the individual constituent moduli. Intuitively, we expect that the basic requirement on a single modulus is the existence of values in the set of couplings $\left\{J_{i, i+1}\right\}$ such that the end qubits in the modulus form a maximally entangled pair (a dimer). This implies a structure of symmetric couplings inside the modulus as, in general, mirror symmetry is a necessary condition for nonvanishing end-to-end entanglement 

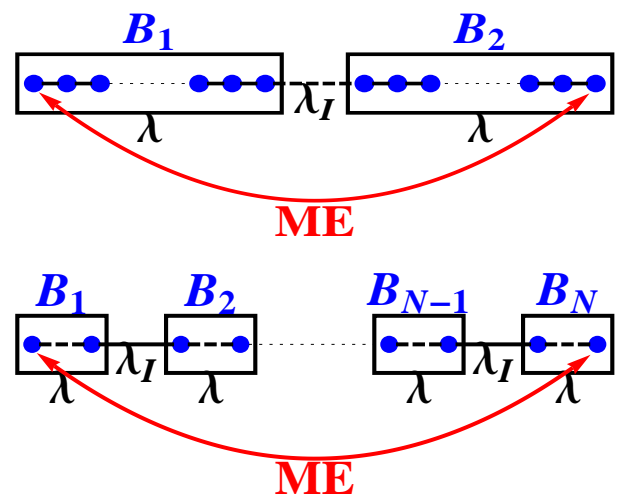

FIG. 1: (color online) Upper panel: A two-moduli system with end-to-end ME. The moduli interact via the local coupling $\lambda_{I}$. Inside each modulus, the bulk is uniformly interacting, whereas $\lambda$ denotes the weak end bond coupling. Lower panel: Generalization $N$ identically interacting moduli.

$[5,7,8]$. As the final yield will be qualitatively independent of the specific realization, we take as benchmark constituent modulus a uniformly interacting system with distorted end bonds: $J_{1,2}=J_{n-1, n}=\lambda J, J_{i, i+1}=J$ for $i \in[2, n-2]$. Fixing $J$ as the unit, one is left with two (dimensionless) free single-modulus parameters: the end bond coupling $\lambda$ and the modulus size $n$.

In Fig. 2 the overall end-to-end bipartite ME in the reduced state of the end qubits 1 and $2 n$, measured by the concurrence $C_{1,2 n}[9]$, is plotted as a function of the remaining free parameter, the modulus-modulus interaction coupling $\lambda_{I}$, for the most elementary modular system composed just of two identical moduli. For comparison, Fig. 22reports also the end-to-end entanglement and the nearest-neighbor (n.n.) end-pair concurrences inside a single modulus, as well as the multipartite entanglement measured by the residual tangle $\tau_{i}^{\text {res }}$ [10]. In Fig. 2 the single-modulus end bond $\lambda$ is such that, for $\lambda_{I}=0$, both the end-to-end entanglement and the end-pair n.n. concurrences are nonvanishing inside the modulus, as shown in the insets. Panel a) shows that as $\lambda_{I}$ crosses a threshold value $\lambda_{I}^{\text {th }}$, the ME is nonvanishing and monotonically increasing up to a saturation value always larger than the end-to-end entanglement originally present in the noninteracting moduli $\left(\lambda_{I}=0\right)$. The threshold coupling $\lambda_{I}^{t h}$ depends on the single-modulus end-to-end entanglement via the single-modulus end bond $\lambda$ and the number $n$ of sites per modulus (see Fig. 31). The rise of $\mathrm{ME}$ is to the detriment of the end-to-end entanglement and end-pair concurrences inside each single modulus. Both rapidly vanish with increasing $\lambda_{I}$. On the other hand, both ME and multipartite entanglement initially increase with $\lambda_{I}$ until, exactly at the crossing of ME with the single-modulus end-to-end entanglement, the residual tangle peaks and then decreases for larger values of $\lambda_{I}$. Therefore, the onset of ME in the ground state of a two-moduli system with an even number of qubits per
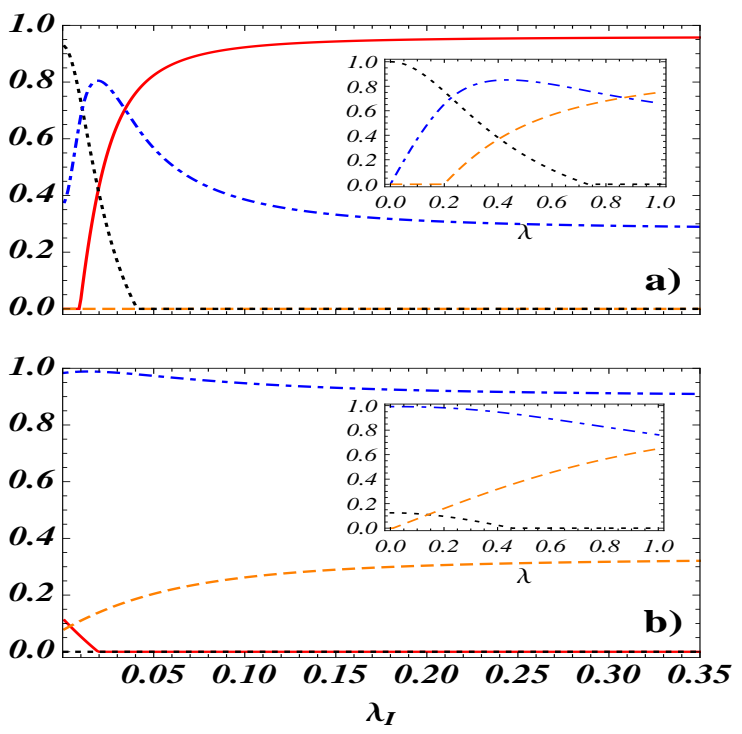

FIG. 2: (color online) Entanglement in a two-moduli spin system. Overall end-to-end $\mathrm{ME} C_{1,2 n}$ (solid red line), end-to-end entanglement inside a single modulus $C_{1, n} \equiv C_{n+1,2 n}$ (dotted black line), end-pair concurrence $C_{1,2} \equiv C_{2 n-1,2 n}$ (dashed orange line), and multipartite entanglement $\left(\tau_{1}^{\text {res }}\right)^{1 / 2}$ (dotdashed blue line), as functions of the inter-modulus coupling $\lambda_{I}$, at single-modulus end bond $\lambda=0.1$. Panel a): each modulus with $n=6$ sites. Panel b): each modulus with $n=7$ sites. Insets: $C_{1, n} \equiv C_{n+1,2 n}$ (dotted black line), $C_{1,2} \equiv C_{2 n-1,2 n}$ (dashed orange line), and $\left(\tau_{1}^{\text {res }}\right)^{1 / 2}$ (dotdashed blue line) inside noninteracting moduli $\left(\lambda_{I}=0\right)$, all as functions of the single-modulus end bond $\lambda$.

modulus is due to the conversion of all forms of bipartite entanglement, originally present in the non-interacting moduli, into ME and, perhaps even more strikingly, the partial conversion into ME of the ground-state multipartite entanglement. Viceversa, no ME is created in a two-

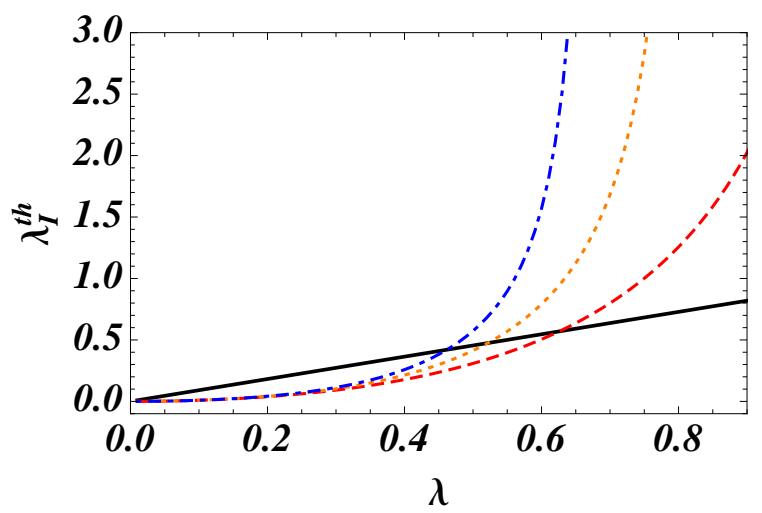

FIG. 3: (color online) Inter-modulus threshold coupling $\lambda_{I}^{t h}$ for the onset of ME as a function of the single-modulus end bond $\lambda$ in two-moduli systems with a number of sites per modulus $n=2$ (solid black line), $n=4$ (dashed red line), $n=6$ (dotted orange line), and $n=8$ (dot-dashed blue line). 


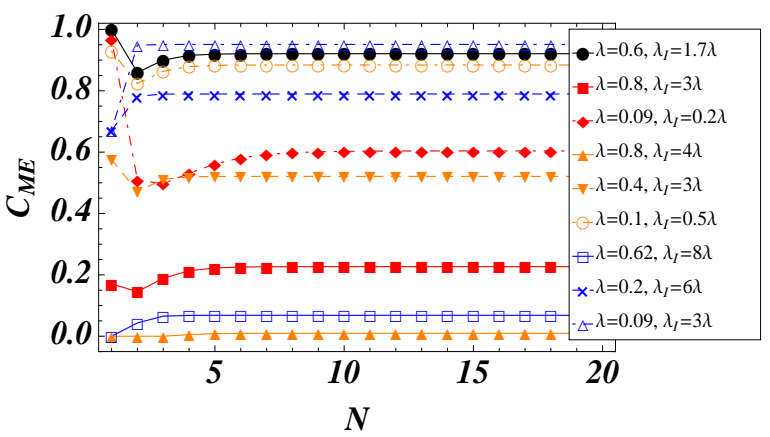

FIG. 4: (color online) End-to-end concurrence, for different values of the intra-modulus and inter-modulus couplings $\lambda$ and $\lambda_{I}$, as a function of the number of moduli $N$ in systems with a number of qubits per modulus $n=2$ (black full circles), $n=4$ (red full squares and diamonds), $n=6$ (orange full triangles and inverted triangles, and empty circles), and $n=$ 8 (blue empty triangles and squares, and dashed line). We consider systems up to $n_{t}=160$ qubits, corresponding to $N=20$ moduli and $n=8$ qubits per modulus.

moduli system with an odd number of qubits per modulus, due to the impossibility of suppressing multipartite correlations inside each modulus, as shown in panel b) of Fig. 21). The presence of a region where end-to-end entanglement prevails over multipartite entanglement inside a noninteracting modulus emerges as the distinctive feature for the presence of ME in a two-moduli system.

Many moduli: We now investigate systems made of an arbitrary number of interacting moduli. We will show that such systems exhibit genuine ME, i.e. sizeindependent, nonvanishing end-to-end entanglement. We

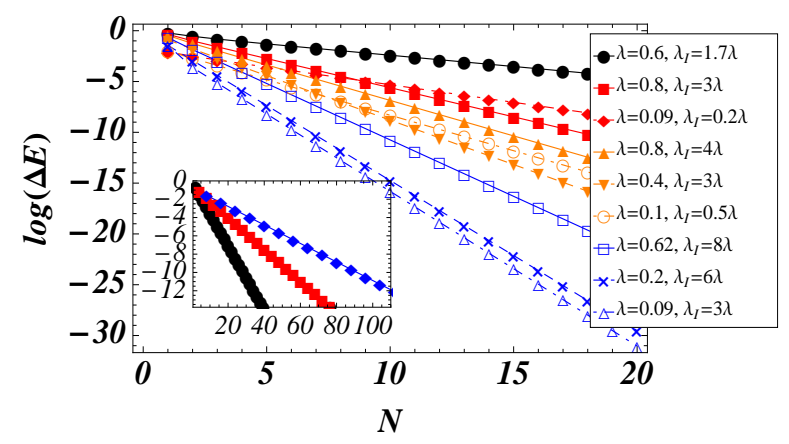

FIG. 5: (color online) Energy gap, for different values of the intra-modulus and inter-modulus couplings $\lambda$ and $\lambda_{I}$, as a function of the number of moduli $N$ in systems with a number of spins per modulus $n=2$ (black full circles), $n=4$ (red full squares and diamonds), $n=6$ (orange full triangles and inverted triangles, and empty circles), and $n=8$ (blue empty triangles and squares, and dashed line). Inset: energy gap as a function of the total number of sites $N n$ in systems with a number of spins per modulus $n=2$ (black full circles), $n=4$ (red full squares), and $n=8$ (blue full diamonds). As in Fig. 4 we consider systems up to $n_{t}=160$ spins.

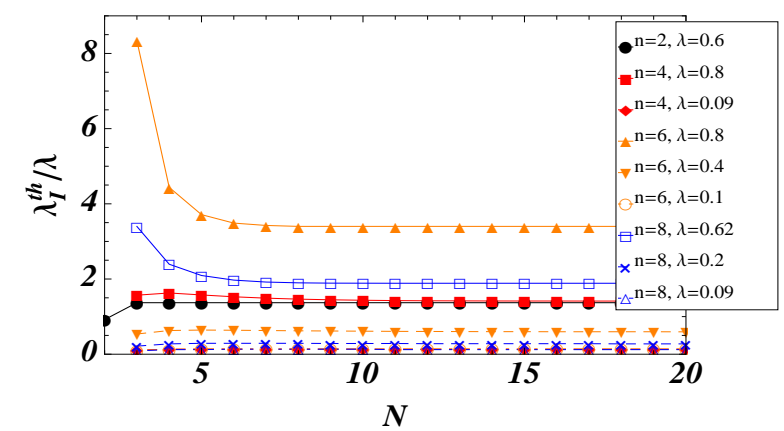

FIG. 6: (color online) Modulus-modulus threshold coupling $\lambda_{I}^{t h}$ for the onset of ME as a function of the number of moduli $N$, in a system with a number of spins per modulus $n=2$ (black full circles), $n=4$ (red full squares and diamonds), $n=6$ (orange full triangles and inverted triangles), and $n=8$ (blue empty triangles and squares, and dashed line). As in Fig. 4 we consider systems up to $n_{t}=160$ spins.

consider the class of Hamiltonians:

$$
\sum_{k=1}^{N} H_{(k-1) n+1, k n}+\sum_{k=1}^{N-1} H_{k n, k n+1}^{I},
$$

where $H_{(k-1) n, k n}$, the Hamiltonian of the $k$ th constituting modulus replica, and $H_{n k, n k+1}^{I}$, the interaction Hamiltonian between the $k$ th and the $(k+1)$ th replicas, are both of the form Eq. (2), $N$ is the number of moduli and $\lambda_{I}$ is the inter-modulus coupling. The main result of the following analysis will be that whenever a quantum system exhibits a structure as that of Eq. (3), then the two end-site elementary subsystems tend to form a long-distance maximally entangled pair in the ground state. The ME expressed by the end-to-end concurrence

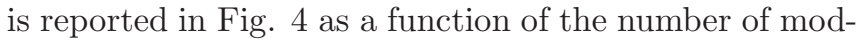
uli $N$, for different values of the inter-modulus coupling $\lambda_{I}$, the number of qubits per modulus $n$, and the intramodulus coupling $\lambda$. The data clearly show the onset of $\mathrm{ME}$ as well as its fast convergence to an asymptotic value. It is important to observe that, depending on the value of $\lambda_{I}$, the generated ME can be either larger or smaller compared to the pre-existing end-to-end entanglement inside a non-interacting modulus. Remarkably, even starting from individual moduli with vanishing endto-end entanglement, there exist intervals of values of $\lambda_{I}$ such that a nonvanishing end-to-end ME is created by means of a collective interaction among the moduli (see e.g. the curve for a system made of six-qubit moduli with $\lambda=0.8, \lambda_{I}=4 \lambda$ in Fig. (4). Moreover, the rate of convergence to the asymptotic value of ME depends essentially on the size of the constituent moduli. The smaller the constituent moduli, the larger the number of moduli that are needed to reach it. Furthermore (see also the discussion below), ME is generated also if $\lambda_{I} / \lambda<1$, at variance with the case of LDE. Indeed, in the latter case the end bonds must always be weakest [4, [5]. Fi- 
nally, ME arises due to the interaction between moduli rather than between individual qubits. This becomes apparent from Fig. 5. where the energy gap is plotted as a function of the number of moduli. In fact, the characteristic exponentially decreasing gap behavior of $\mathrm{ME}$ is clearly dependent on the number of moduli and not on the number of sites. In the inset of Fig. 5 the gap is plotted as a function of the number of sites for modular systems with different numbers of sites per modulus, in the same correlation regime. Given the same total number of qubits, the energy gap in a modular system with $2 n$ sites per modulus is approximately given by the square root of the gap in a modular system with $n$ sites per modulus, and so on. Therefore the ground-state ME in a modular system is much more robust against temperature than the corresponding LDE counterpart, with an improvement in the gap that can reach some orders of magnitude. Figs. 3 and 4 clarify the relation between the onset of $\mathrm{ME}$ and the inter-modulus coupling $\lambda_{I}$. Whereas a nonvanishing LDE inside a single modulus generally requires a constraint on the relative ratios of the couplings within the modulus, the collective interaction leading to the onset of $\mathrm{ME}$ does not. Comparing how ME arises in systems made of moduli of equal size $n$ but different values of $\lambda$, there emerges the existence of a threshold value of $\lambda_{I}^{t r}$ which can be either larger or smaller than the intra-modulus coupling $\lambda$. In Fig. 6] we plot the ratio $\lambda_{I}^{t h} / \lambda$ as a function of the number of moduli $N$, for moduli with different $n$ and $\lambda$. By examining the curves at fixed $n$, we observe three different behaviors of $\lambda_{I}^{t r}$, according to the different intra-modulus correlation regimes. Namely, if the end-to-end entanglement inside each modulus is either vanishing or nearly vanishing, then the thermodynamic limit is monotonically approached from above and $\lambda_{I} / \lambda \gg 1$; in the opposite regime, i.e. for maximal end-to-end entanglement inside the individual moduli, the limit is monotonically approached from below with $\lambda_{I} / \lambda \ll 1$. In the intermediate regime, we observe an almost constant averaging behavior of $\lambda_{I}^{t h}$ in the neighborhood of $\lambda$, with amplitude oscillations that slow down in the proximity of the thermodynamic value. Systems composed by minimal two-qubit moduli, i.e. those exhibiting LDE, have thus a rather pathological behavior due to the bare intra-modulus entanglement being always maximal regardless of the value of $\lambda$. Namely, the ratio $\lambda_{I} / \lambda$ must always be larger than unity and acquire the same for every $\lambda$.

Summing up, the existence of large end-to-end ME relies on a mechanism of balancing/compensation between the bipartite and the multipartite entanglement present in a system, as well as on the form of the energy spectrum and the degree of modularity that can be engineered in the system. One of the most important practical advantages of $\mathrm{ME}$ over $\mathrm{LDE}$ is that the energy gap scales exponentially with the total number of moduli, rather than with the total number of qubits. This difference implies that, given a fixed total number of qubits in the system, the stability of ME against thermal noise can be enhanced by orders of magnitude, compared to LDE, just by a proper tailoring of the number of moduli and of the single-modulus parameters, as shown in Fig. 5 above. Moreover, being a static, ground-state property, there is no question on the time-scale and speed at which ME can be created. These two aspects (enhanced thermal stability and exact ground state property) of ME are crucial advantages over schemes that suggest to create entanglement between distant qubits dynamically by, e.g., a sudden global quench much below a certain threshold temperature [11]. Indeed, dynamical schemes are intrinsically fragile to noise, imperfections, and thermal fluctuations. Moreover, they decay quickly with the size of the system. On the contrary, ME is a size-independent, exact ground-state property resilient to thermal decoherence and robust against imperfections. Its experimental realization and control would thus provide a relevant step towards the implementation of faithful largescale quantum teleportation, memories, and repeaters, as well as the preparation of large multi-partite states for measurement-based quantum computation.

Acknowledgements: We acknowledge financial support from the European Union under the FP7 STREP Project HIP (Hybrid Information Processing), Grant Agreement No. 221889.

[1] M. A. Nielsen and I. L. Chuang, Quantum Computation and Quantum Information (Cambridge University Press, Cambridge, 2000).

[2] L. Amico, R. Fazio, A. Osterloh, and V. Vedral, Rev. Mod. Phys. 80, 517 (2008).

[3] V. Coffman, J. Kundu, and W. K. Wootters, Phys. Rev. A 61, 052306 (2000); T. J. Osborne and F. Verstraete, Phys. Rev. Lett. 96, 220503 (2006).

[4] L. Campos Venuti, C. Degli Esposti Boschi, and M. Roncaglia, Phys. Rev. Lett. 96, 247206 (2006); L. Campos Venuti, S. M. Giampaolo, F. Illuminati, and P. Zanardi, Phys. Rev. A 76, 052328 (2007).

[5] S. M. Giampaolo and F. Illuminati, New J. Phys. 12, 025019 (2010).

[6] S. M. Giampaolo and F. Illuminati, Phys. Rev. A 80, 050301 (2009).

[7] C. Albanese, M. Christandl, N. Datta, and A. Ekert, Phys. Rev. Lett. 93, 230502 (2004).

[8] G. Gualdi, I. Marzoli, and P. Tombesi, New J. Phys. 11, 063038 (2009).

[9] W. K. Wootters, Phys. Rev. Lett. 80, 2245 (1998).

[10] W. Dür, G. Vidal, and J. I. Cirac, Phys. Rev. A 62, 062314 (2000); T. Roscilde, P. Verrucchi, A. Fubini, S. Haas, and V. Tognetti, Phys. Rev. Lett. 93, 167203 (2004).

[11] H. Wichterich and S. Bose, Phys. Rev. A 79, 060302(R) (2009). 